\title{
The modelling of the X-ray emission of planetary nebulae
}

\author{
D. Schönberner, M. Steffen and A. Warmuth \\ Astrophysikalisches Institut Potsdam, 14482 Potsdam, Germany \\ email: deschoenberner@aip.de, msteffen@aip.de, awarmuth@aip.de
}

\begin{abstract}
We report on our recent efforts to compute the X-ray emission from the hot, shocked stellar wind gas filling the inner cavity of planetary nebulae. To this end, we updated our 1D hydrodynamics code NEBEL by including a module that computes the heat transfer by thermal conduction across the contact discontinuity separating the hot shocked wind gas from the much cooler nebular material. Given the temperature and density structure of the hot bubble the X-ray emission is computed by means of the CHIANTI code. We find a reasonably close agreement of the computed X-ray luminosities with recent observations of Newton-XMM and Chandra. Our simulations also predict how the X-ray emission depends on the wind luminosity and the stellar parameters.
\end{abstract}

Keywords. hydrodynamics - radiative transfer - planetary nebulae:general - X-rays:stars

\section{1. introduction}

The modern, very successful concept for the formation and evolution of the main structures of planetary nebulae $(\mathrm{PNe})$ is based on the dynamical effects caused by (i) the heating by photoionization of the red-giant wind material ejected earlier and (ii) the interaction of a time-dependent central-star wind with the ambient ionized shell. For a recent review see Schönberner \& Steffen (2003). The wind from the central star is very fast, with typically a few $1000 \mathrm{~km} \mathrm{~s}^{-1}$, and passes through a shock before it encounters the dense slow wind. A tenuous but very hot $\left(\gtrsim 10^{7} \mathrm{~K}\right)$ 'bubble' is formed since $9 / 16$ of the mechanical wind energy is converted into internal energy behind the shock. The bubble is separated from the outer, much cooler nebular gas by a contact discontinuity.

The shocked wind gas including the thin transition regime towards the PN proper are the likely sites from which one has to expect diffuse X-ray emission. The detection of diffuse X-ray emission from PNe is a direct confirmation of the existence of hot shocked gas, predicted theoretically as a consequence of the high-speed central-star wind (Kwok et al. 1978). An extensive summary of all the X-ray observations available to date is presented by Guerrero (these proceedings). Although the detection of shocked wind material was gratifying, the properties of the X-ray emitting gas were disturbing. It turned out that the X-ray spectra are rather soft, indicating temperatures of only $\approx 2 \ldots 3 \times 10^{6} \mathrm{~K}$. The electron densities of the emitting volumes vary between about 20 and $200 \mathrm{~cm}^{-3}$. These results are in sharp contrast to theoretical expectations since the observed wind velocities and mass-loss rates demand bubble temperatures of $\approx 10^{7} \ldots 10^{8} \mathrm{~K}$ and electron densities far below the observed ones.

\section{The modelling}

Already Weaver et al. (1977) and later Soker (1994) argued that heat conduction by electrons across the interface between the hot shocked gas and the much cooler 
environment must be considered. To date, theoretical estimates of the X-ray emission from planetary nebulae are based on analytic, self-similar solutions of the bubble structure with power-law stellar evolution (Zhekov \& Perinotto 1996, 1998). We thus decided to to implement a heat conduction module into our $1 \mathrm{D}$ radiation-hydrodynamics code NEBEL, a detailed description of which can be found in Perinotto et al. (2004).

Heat conduction by electrons is described as a diffusion process, with the heat flux $\vec{q}$ given by

$$
\vec{q}=-D \nabla T_{\mathrm{e}}
$$

Following Spitzer (1962) and Cowie \& McKee (1977), the electron mean free path $\lambda$ can be written as

$$
\lambda=2.625 \cdot 10^{5} T_{\mathrm{e}}^{2} / n_{\mathrm{e}} / \ln \Lambda \quad[\mathrm{cm}]
$$

where the Coulomb Logarithm $\ln \Lambda$ can be approximated as

$$
\ln \Lambda=\left\{\begin{array}{lll}
9.425+3 / 2 & \ln T_{\mathrm{e}}-1 / 2 \ln n_{\mathrm{e}}, & T_{\mathrm{e}} \leqslant 4.2 \cdot 10^{5} \mathrm{~K} \\
22.37+ & \ln T_{\mathrm{e}}-1 / 2 \ln n_{\mathrm{e}}, & T_{\mathrm{e}}>4.2 \cdot 10^{5} \mathrm{~K}
\end{array}\right.
$$

for a pure hydrogen plasma. The diffusion coefficient $D$ is then given by

$$
D=7.04 \cdot 10^{-11} \lambda n_{\mathrm{e}} T_{\mathrm{e}}^{1 / 2} \quad[\mathrm{erg} / \mathrm{s} / \mathrm{K} / \mathrm{cm}] .
$$

At high $T_{\mathrm{e}}$ and low $n_{\mathrm{e}}$, the electron mean free path $\lambda$ becomes very large according to Eq. (2.2) (actually it can become much larger than the dimensions of the 'hot bubble'), and the diffusion approximation is no longer valid. Rather, the heat flux cannot exceed the saturation limit

$$
\vec{q}_{\mathrm{sat}}=1.72 \cdot 10^{-11} T_{\mathrm{e}}^{3 / 2} n_{\mathrm{e}} \quad\left[\mathrm{erg} / \mathrm{cm}^{2} / \mathrm{s}\right] .
$$

This is an approximate upper limit expressing the fact that the heat flux cannot be larger than the heat content, $3 / 2 n_{\mathrm{e}} k T_{\mathrm{e}}$, times a characteristic electron transport velocity, $v_{\text {char }}$. For a more detailed derivation of Eq. (2.5) see Cowie \& McKee (1977).

To compute the synthetic X-ray spectra we employed the well-documented CHIANTI software package (Dere et al. 1997), version 5.1 which includes the most recent atomic data (Landi et al. 2006). Only radial shells with $T_{\mathrm{e}}>10^{5} \mathrm{~K}$ were considered since the contribution of cooler layers is negligible to the X-ray luminosity. With the input of $T_{\mathrm{e}}, n_{\mathrm{e}}$ and $n_{\mathrm{p}}$ for a given volume element, individual spectra are then synthesized, including the contribution due to lines and various continua (free-free, free-bound and two-photon continuum). We have used the ion fractions from Mazzotta et al. (1998), under the assumption of ionization equilibrium.

Each individual spectrum actually represents the emission (in $\mathrm{erg} \mathrm{cm}^{-3} \mathrm{~s}^{-1} \AA^{-1}$ ) from a unit volume. In order to determine the total X-ray emission, the averaged spectrum, and the brightness distribution on the plane of the sky, we must perform appropriate integrations over our spectra, taking the (spherical) geometry of the model into account. The multiplication of an individual spectrum with the volume of the corresponding radial shell gives the total emission from that shell, and the summation over all shells emitting in X-rays then yields the total spectrum. An integration over a certain wavelength range then results in a total X-ray luminosity, $L_{\mathrm{X}}$ (in $\operatorname{erg~s}^{-1}$ ).

For determining the brightness distribution in the plane of sky (in $\operatorname{erg} \mathrm{cm}^{-2} \mathrm{~s}^{-1} \mathrm{sr}^{-1}$ ), the integration is performed along the line of sight (perpendicular to the plane of sky) for a series of impact parameters (200).

The computation of the complete X-ray spectrum and luminosity is rather time consuming and thus only performed for selected models along an evolutionary sequence. Such a post facto computation of the X-ray emission is somewhat inconsistent with the 
hydrodynamics since part of the energy lost from the bubble by X-rays is not included in the energy budget. We note, however, that the total X-ray luminosity is only a very small fraction of the wind power $\left(\simeq 10^{-3}\right.$, see next Sect.), and an incomplete consideration of the X-ray emission in the bubble's energy budget has virtually no consequences for the dynamics of the whole system.

\section{First results}

The strong influence of heat conduction across the contact surface is illustrated in Fig. 1. While the size of the bubble remains virtually the same, its properties are very different: with heat conduction the density is more than 10 times larger, the (electron) temperature correspondingly lower, with values well below $10^{7} \mathrm{~K}$. The amount of material in the bubble is dominated by nebular gas heated and evaporated by the heat conduction front. Large parts of the bubble emit soft X-rays, generating a luminosity about $100 \times$ larger compared with the model without heat conduction. Note that the central-star wind remains cool, and that the PN proper is also not affected by heat conduction.

Our X-ray luminosities appear to be somewhat smaller than those computed by Zhekov \& Perinotto $(1996,1998)$, but they are in fair agreement with recent observations (Guerrero, priv. comm.), as demonstrated in Fig. 2. There we have plotted only luminosity ratios to minimize errors based on incorrect distances. For most of the evolution, the X-ray luminosity increases with time. Generally, the models predict that only a very tiny amount of the central star wind power is converted into X-rays (right panel of Fig. 2), and that this fraction remains rather constant for most of the PN's lifetime. More massive central stars generate larger X-ray luminosities via their more vigorous winds. Below an effective temperature of about $20000 \mathrm{~K}$ the stellar wind is too weak to provide shocked gas with the required temperature of at least $10^{6} \mathrm{~K}$. Likewise, the X-ray luminosity drops when the central star fades towards the white-dwarf configuration.
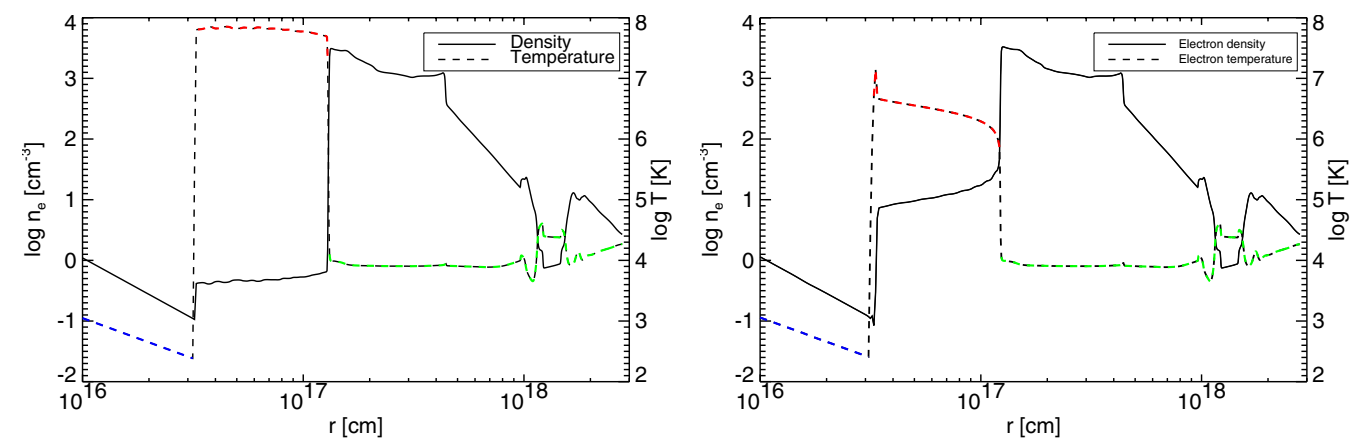

Figure 1. Radial profiles of electron densities (solid) and temperatures (dashed) of two models at about the same position along the evolutionary tracks of a $0.595 \mathrm{M}_{\odot}$ central star, one without (left) and one with heat conduction (right), computed under otherwise identical conditions. The stellar parameters are: $L \simeq 5200 \mathrm{~L}_{\odot}$ and $T_{\text {eff }} \simeq 71568 \mathrm{~K}$ at an age of $t \simeq 5640 \mathrm{yr}$. The central star is at the origin, and the wind shock at $r \simeq 3 \times 10^{16} \mathrm{~cm}$. The PN proper is (in both cases) bounded by a contact surface $\left(r \simeq 1.3 \times 10^{17} \mathrm{~cm}\right)$ and an outer shock front at $r \simeq 4.4 \times 10^{17} \mathrm{~cm}$. The PN is surrounded by the ionized AGB wind whose radial density profile reflects the mass-loss history of the late AGB evolution (cf. Steffen et al. 1998). 

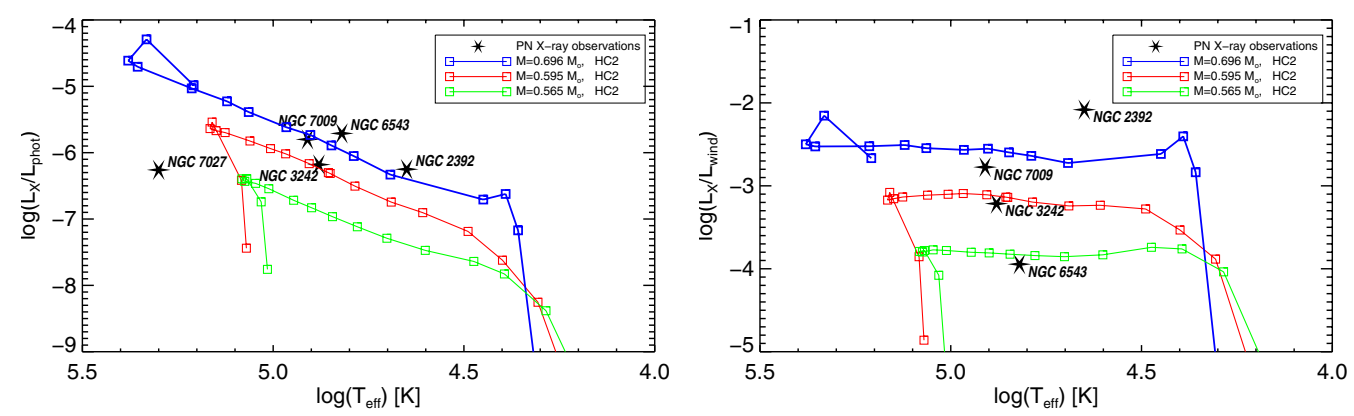

Figure 2. Left: X-ray luminosity of the bubble in the $0.45-2.5 \mathrm{keV}(5-28 \AA)$ energy range over stellar photon luminosity as a function of the stellar effective temperature, for model sequences with different central stars as indicated in the inset. The observations are from Guerrero (priv. comm.). Only objects with hydrogen-rich central stars are used in this figure. Right: X-ray luminosity of the bubble over stellar wind power as a function of the stellar effective temperature for the same sequences and observations as in the left panel. NGC 7027 is not shown because of its unknown wind parameters.

\section{Conclusion and outlook}

Our hydrodynamical simulations including heat conduction allow for the first time a realistic computation of the X-ray emission from the hot, shocked wind gas enclosed by the PN proper as a function of the evolution across the H-R diagram. In the future it is important to simulate the evolution of nebular models around hydrogen-poor central stars where one expects X-ray emission from hydrogen-poor, carbon-rich bubble gas. A detailed analysis of high-resolution X-ray spectra from PNe with WC central stars may allow to locate the chemical discontinuity, which in turn would give constraints for the origin of the hydrogen deficiency. A further challenge is the consideration of magnetic fields which will suppress heat conduction except in the direction of the field lines (cf. Borkowski et al. 1990).

\section{References}

Borkowski, K.J., Balbus, S.A. \& Fristrom, C.C. 1990, ApJ, 355, 501

Cowie, L.L. \& McKee, C.F. 1977, ApJ 211, 135

Dere, K.P., Landi, E., Mason, H.E., Monsignori Fossi, B.C. \& Young, P.R. 1997, A $\mathscr{G} A S, 125$, 149

Kwok, S., Purton, C.R. \& FitzGerald, P.M. 1978, ApJ 219, L125

Landi, E., Del Zanna, G., Young, P.R., Dere, K.P., Mason, H.E. \& Landini, M. 2006, ApJS 160, 261

Mazzotta, P., Mazzitelli, G., Colafrancesco, S. \& Vittorio, N. 1998, A\&AS 133, 403

Perinotto, M., Schönberner, D., Steffen, M. \& Calonaci, C. 2004, A $\mathscr{S} A$ 414, 993

Schönberner, D. \& Steffen, M. 2003, in: S. Kwok, M. Dopita, \& R. Sutherland (eds.), Planetary Nebulae: Their Evolution and Role in the Universe, IAU Symp. 209, p. 147

Soker, N. 1994, AJ 107, 276

Spitzer, L. 1962, Physics of Fully Ionized Gases, 2nd rev. ed., Wiley Interscience Publishers

Steffen, M., Szczerba, R. \& Schönberner, D. 1998, A\&A 337, 149

Weaver, R., McCray, R., Castor, J., Shapiro, P. \& Moore, R. 1977, ApJ 218, 377

Zhekov, S.A. \& Perinotto, M. 1996, A\&SA 309, 648

Zhekov, S.A. \& Perinotto, M. 1998, A\&A 334, 239 